\title{
A first order wavefront estimation algorithm for P1640 calibrator
}

\author{
C. Zhai ${ }^{a}$, G. Vasisht ${ }^{a}$, M. Shao ${ }^{a}$, T. Lockhart ${ }^{a}$, E. Cady $^{a}$, \\ B. Oppenheimer ${ }^{b}$, R. Burruss ${ }^{a}$, J. Roberts ${ }^{a}$, \\ C. Beichman ${ }^{c}$, D. Brenner ${ }^{b}$, J. Crepp $^{c}$, R. Dekany ${ }^{c}$,

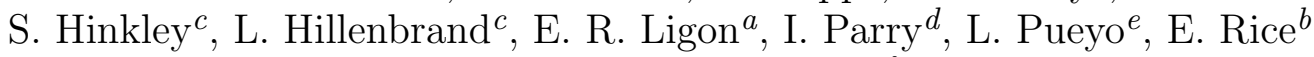 \\ L. C. Roberts Jr. ${ }^{a}$, A. Sivaramakrishnan ${ }^{f}$, R. Soummer ${ }^{e}$ \\ F. Vescelus ${ }^{a}$ K. Wallace $^{a}$ N. Zimmerman ${ }^{g}$, \\ ${ }^{a}$ Jet Propulsion Laboratory, California Institute of Technology, \\ 4800 Oak Grove Dr, Pasadena, CA, 91109; \\ ${ }^{b}$ American Museum of Natural History, Central Park West at 79th Street, New York, NY \\ 10024; \\ ${ }^{c}$ California Institute of Technology, 1200 E. California Blvd., Pasadena, CA 91125; \\ ${ }^{d}$ Institute of Astronomy, Cambridge University; \\ ${ }^{e}$ Johns Hopkins University, Physics and Astronomy Dept., Baltimore, MD 21218 USA; \\ ${ }^{f}$ Space Telescope Science Institute, Baltimore, MD 21218, USA; \\ ${ }^{g}$ Max Planck Institute for Astronomy, Heidelberg, Germany
}

\begin{abstract}
P1640 calibrator is a wavefront sensor working with the P1640 coronagraph and the Palomar 3000 actuator adaptive optics system (P3K) at the Palomar 200 inch Hale telescope. It measures the wavefront by interfering post-coronagraph light with a reference beam formed by low-pass filtering the blocked light from the coronagraph focal plane mask. The P1640 instrument has a similar architecture to the Gemini Planet Imager (GPI) and its performance is currently limited by the quasi-static speckles due to non-common path wavefront errors, which comes from the non-common path for the light to arrive at the AO wavefront sensor and the coronagraph mask. By measuring the wavefront after the coronagraph mask, the non-common path wavefront error can be estimated and corrected by feeding back the error signal to the deformable mirror (DM) of the P3K AO system. Here, we present a first order wavefront estimation algorithm and an instrument calibration scheme used in experiments done recently at Palomar observatory. We calibrate the P1640 calibrator by measuring its responses to poking DM actuators with a sparse checkerboard pattern at different amplitudes. The calibration yields a complex normalization factor for wavefront estimation and establishes the registration of the DM actuators at the pupil camera of the P1640 calibrator, necessary for wavefront correction. Improvement of imaging quality after feeding back the wavefront correction to the AO system demonstrated the efficacy of the algorithm. (C) Copyright 2012. All rights reserved.
\end{abstract}

\section{INTRODUCTION}

High contrast imaging is a powerful way to detect and characterize exoplanets. The quality of high contrast images depends critically on the quality of wavefront. This is especially true for coronagraphy, which is designed for a nominal ideal wavefront. To achieve a wavefront with low aberrations, the P1640 project uses the state of art Palomar 3000 actuator adaptive optics (P3K AO) to actively compensate wavefront errors due to atmosphere as well as the imperfect optics in the telescope. While the high contrast imaging system relies crucially on the performance of the $\mathrm{AO}$ system, a perfect $\mathrm{AO}$ system, however, does not guarantee best performance of the coronagraphy because the $\mathrm{AO}$ system controls the wavefront to minimize the wavefront error at the wavefront

Further author information: (Send correspondence to C.Z.)C.Z.: E-mail: chengxing.zhai@jpl.nasa.gov, Telephone: 1 8183930758

Adaptive Optics Systems III, edited by Brent L. Ellerbroek, Enrico Marchetti, Jean-Pierre Véran, Proc. of SPIE Vol. 8447, 84476W · @ 2012 SPIE · CCC code: $0277-786 / 12 / \$ 18 \cdot$ doi: $10.1117 / 12.927015$ 


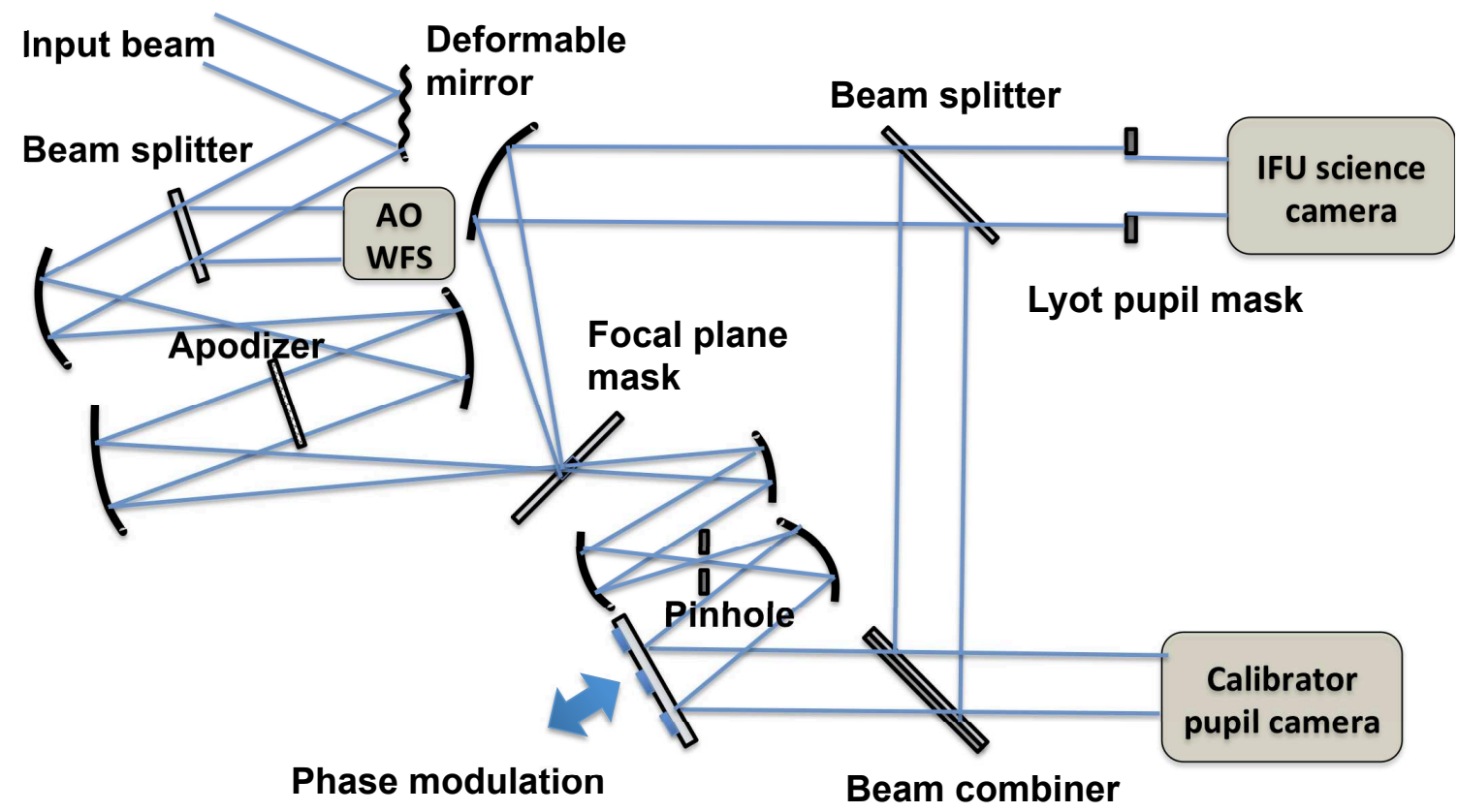

Figure 1. A functional sketch of the P1640 high contrast imaging system, which includes the P3K AO system, P1640 Apodized Lyot Coronagraph system, and the P1640 Calibrator.

sensor of the AO system while the performance of the coronagraph imaging system is mainly determined by the quality of the wavefront at just before the coronagraph mask. It is this differential paths for the light to propagate to the $\mathrm{AO}$ wavefront sensor and to the coronagraph mask that produces the non-common path error. The non-common path wavefront errors lead to quasi-static speckles that is currently limiting the performance of P1640 coronagraph.

To sense the non-common path error, P1640 Calibrator, a wavefront sensor based on Michelson interferometry, has been developed at the Jet Propulsion Laboratory. It measures the wavefront by interfering post-coronagraph E-field with a reference beam formed by low-pass filtering the light blocked by the coronagraph mask. ${ }^{1}$ Fig. 1 shows a functional sketch of the P1640 high contrast imaging system, which uses an architecture similar to that of the Gemini Planet Imager (GPI). ${ }^{2}$ For more detailed descriptions of P1640, see references. ${ }^{3,4}$

The input beam can be from the telescope or an internal source for experimenting. It is first reflected by the deformable mirror (DM, at the top left), with part of the light split into the AO wavefront sensor, goes through an apodized pupil mask, and is then focused on to a focal plane coronagraph mask where the "blocked" light leaks through the hole and the reflected light goes to the science instrument, an integral field spectrograph(IFS). ${ }^{2,3}$ The apodized pupil mask tapers the beam amplitude at pupil to maximize the encircled energy in the hole of the focal plane mask and thus improve the coronagraph performance. ${ }^{5}$ Before reaching the IFS, a beam splitter partitions part of the light for the P1640 Calibrator to measure the post-coronagraph E-field using interferometry. We call this beam science beam. The light leaking through the hole of the focal plane coronagraph mask is further lowpass filtered by a pinhole to form a reference beam. The two beams are combined at the beam combiner before entering the Calibrator pupil camera. A phase modulation is introduced to the reference arm for measuring the interference fringes at a pupil after the coronagraph focal plane mask, which we shall call Calibrator pupil, at which the detector of Calibrator pupil camera is located. These fringes are used to estimate the post-coronagraph E-field and to further infer the aberrations before the coronagraph.

In this paper, we describe the algorithms for estimating wavefront using the fringe measurements from the 
P1640 Calibrator. We also describe how we calibrate the P1640 Calibrator to facilitate the wavefront estimation. The P1640 Calibrator uses the conventional ABCD 4-bin staircase modulation to measure the fringes, which are then converted to a map of phasors over the Calibrator pupil. Our instrument calibration estimates the proper normalization for converting the phasor map to an estimate of the wavefront. The estimated wavefront is natively in the Calibrator pupil coordinate, indexed by the Calibrator camera pixels. To feedback a wavefront correction generated based on Calibrator measurements to the DM, we need to register the DM actuators at the Calibrator pupil. We establish this registration by relating the response measured in Calibrator pupil when poking the DM actuators in a sparse checkerboard pattern. An affine relation is then estimated to model the transformation between the DM and Calibrator pupils. The response to sparse checkerboard pattern poking also enables to estimate a low order phase that captures misalignment between the reference science beam because we know the poking pattern is a wavefront pattern in phase (not amplitude). With the proper calibration, we are able to estimate the wavefront aberrations using the fringe measurements. As we iteratively send the derived corrections for compensating the phase errors to the DM in AO system, the estimated wavefront errors get smaller and we observe the improvement of the image quality. This demonstrates the efficacy of the wavefront sensing capability, the estimation algorithm, and the feedback control loop.

\section{BASIC MODEL}

The fringe measurements from the calibrator camera is described by intensity maps $I(x, y)$ labeled by pupil camera pixel index $(x, y)$,

$$
\begin{aligned}
I(x, y) & =\left|E_{\text {ref }}(x, y) \exp (i \theta)+E_{\text {sci }}(x, y)\right|^{2} \\
& =I_{\text {ref }}+I_{\text {sci }}+2 \Re\left\{E_{\text {sci }}(x, y) E_{\text {ref }}(x, y)^{*} \exp (-i \theta)\right\},
\end{aligned}
$$

where $E_{\text {ref }}(x, y)$ and $E_{\text {sci }}(x, y)$ are E-fields at the Calibrator pupil from reference and science beams respectively. The asterisk represents complex conjugate. $\theta$ is the phase introduced by the phase modulation. Intensities of reference and science beams, $I_{\text {ref }}(x, y)$ and $I_{\text {sci }}(x, y)$ are given by

$$
I_{\text {ref }}(x, y)=\left|E_{\text {ref }}(x, y)\right|^{2}, \quad I_{\text {sci }}(x, y)=\left|E_{\text {sci }}(x, y)\right|^{2} .
$$

We adopt a staircase 4-bin modulation to obtain the conventional A,B,C,D measurements ${ }^{6}$ by periodically setting $\theta$ to be $0, \pi / 2, \pi, 3 \pi / 2$. Fig. 2 shows a typical set of four frames measured by the P1640 Calibrator camera. With $\mathrm{A}, \mathrm{B}, \mathrm{C}, \mathrm{D}$ measurements, it is convenient to define phasors $\Phi(x, y)$ as

$$
\Phi(x, y) \equiv \frac{1}{4}[(A-C)+i(B-D)]=E_{\mathrm{sci}}(x, y) E_{\mathrm{ref}}(x, y)^{*},
$$

where the second equality can be justified by plugging in corresponding values of $\theta$ for $\mathrm{A}, \mathrm{B}, \mathrm{C}, \mathrm{D}$ frames in Eq. (1) and carrying out some standard algebraic derivations.

The design of the coronagraph does a high pass filtering in the science beam as the coronagraph mask reflects only light over a disk larger than an angular size of $2.7 \lambda / D$, where $D=5 \mathrm{~m}$ is the diameter of the telescope and $\lambda$ is the effective wavelength at the center of the passband (H-band,about 1650nm) of P1640 Calibrator. Therefore, the science beam E-field $E_{\text {sci }}(x, y)$ contains only the high spatial frequency portion of the input E-field of the coronagraph. It is convenient to use the pupil where DM resides as the input pupil.

To estimate wavefront at the DM pupil, we write the E-field at the DM as

$$
E_{\mathrm{i}}(x, y) \equiv E_{0}\left(1+\epsilon_{i}(x, y)\right) \exp \left[i \phi_{i}(x, y)\right]
$$

$\epsilon_{i}(x, y)$ and $\phi_{i}(x, y)$ are the amplitude and phase errors describing the wavefront aberration. It is convenient to decomposite the amplitude error and phase error according the their spatial frequencies into low $(|\mathbf{k}|<=2.7 / D)$ and high $(|\mathbf{k}|>2.7 / D$ frequency portions:

$$
\epsilon_{\mathrm{i}}(x, y) \equiv \epsilon_{\mathrm{l}}(x, y)+\epsilon_{\mathrm{h}}(x, y), \quad \phi_{\mathrm{i}}(x, y) \equiv \phi_{\mathrm{l}}(x, y)+\phi_{\mathrm{h}}(x, y),
$$



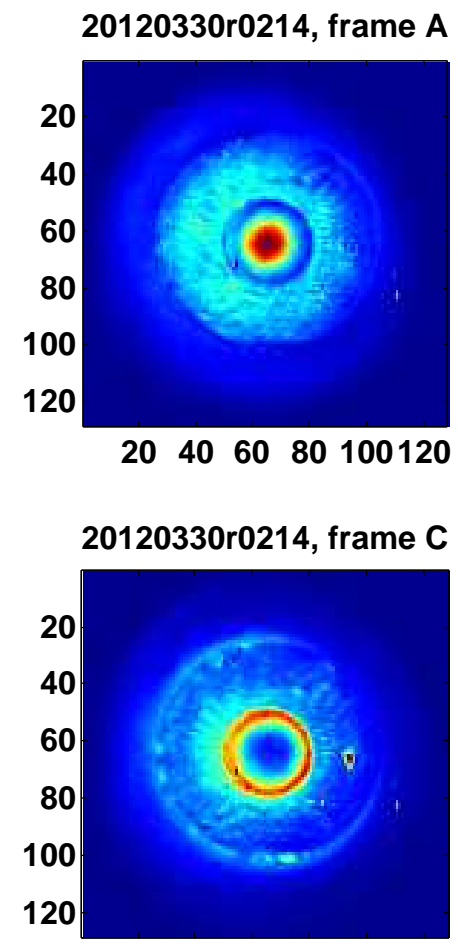

20406080100120
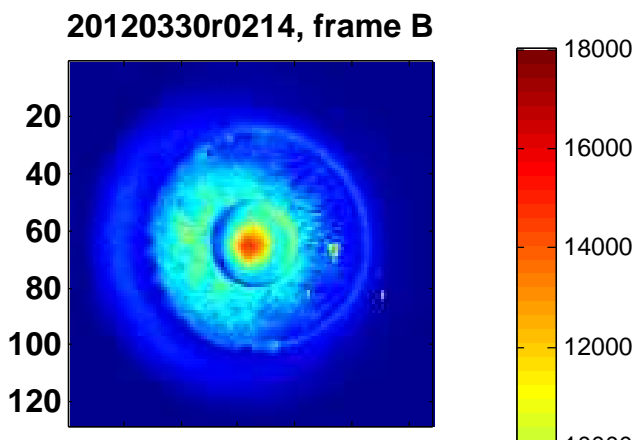

20406080100120

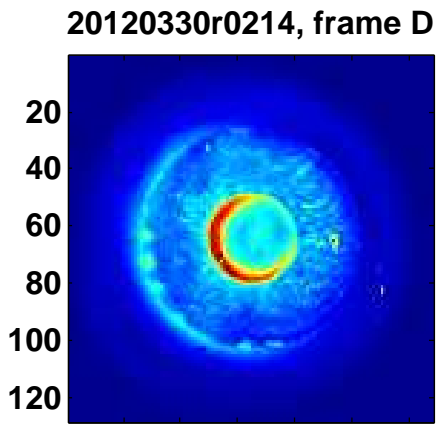

20406080100120

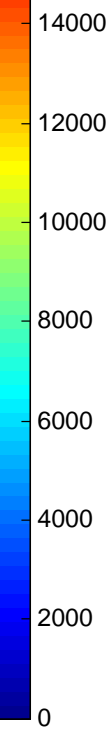

14000

2000

1000

00

000

000

000

Figure 2. Four $128 \times 128$ frames corresponding to the standard A, B, C, D 4-bin fringe measurements. top left (frame A), top right (frame B), bottom left (frame C), bottom right (frame D).

where $\mathbf{k} \equiv\left(k_{x}, k_{y}\right)$ is the spatial frequency vector. The frequency separation boundary corresponds to the filtering cutoff of the focal plane mask of the coronagraph. We also assume that the wavefront errors are much smaller 1 after the AO correction. To the first order,

$$
\begin{aligned}
E_{\mathrm{i}}(x, y) & =E_{0}\left(1+\epsilon_{\mathrm{l}}(x, y)+\epsilon_{\mathrm{h}}(x, y)\right) \exp \left[i \phi_{\mathrm{l}}(x, y)+i \phi_{\mathrm{h}}(x, y)\right] \\
& \approx E_{0}\left(1+\epsilon_{\mathrm{l}}(x, y)+\epsilon_{\mathrm{h}}(x, y)+i \phi_{\mathrm{h}}(x, y)\right) \exp \left[i \phi_{\mathrm{l}}(x, y)\right]
\end{aligned}
$$

After going through the coronagraph, the E-field landing at the P1640 calibrator pupil camera may be modeled as

$$
E_{\mathrm{sci}}(x, y) \approx t_{s} E_{0} A(x, y)\left(\epsilon_{\mathrm{h}}(x, y)+i \phi_{\mathrm{h}}(x, y)\right) \exp \left[i \phi_{1}(x, y)\right]
$$

where we have dropped the low order term in the E-field to model the effect of the effect of the focal plane mask, $t_{s}$ is a multiplicative factor representing the throughput from the DM pupil to the calibrator pupil, and $A(x, y)$ is the transmission function applied at the pupil apodizer. See Fig. 1.

The reference arm E-field is close to a plane wave because it is low pass filtered by a pinhole of diameter $1.4 \lambda / D$. The variation scale of the reference beam E-field is inversely proportional to the size of the pinhole, which is the diameter of the pupil. Fig. 3 shows a typical map of the reference beam intensity $I_{\text {ref }}(x, y)$ measured by closing the shutter to block the science beam. The reference beam intensity measurement gives the magnitude of the reference E-field. It is convenient to introduce a low order differential phase term $\phi_{\mathrm{rs}}(x, y)$ to capture the slowly varying phase in the reference E-field relative to an ideal science beam.

$$
E_{\text {ref }}(x, y)=\sqrt{I_{\text {ref }}(x, y)} \exp \left[i \phi_{\text {rs }}(x, y)\right] .
$$

Note that $\phi_{\mathrm{rs}}(x, y)$ captures misalignment between reference and science beams. 


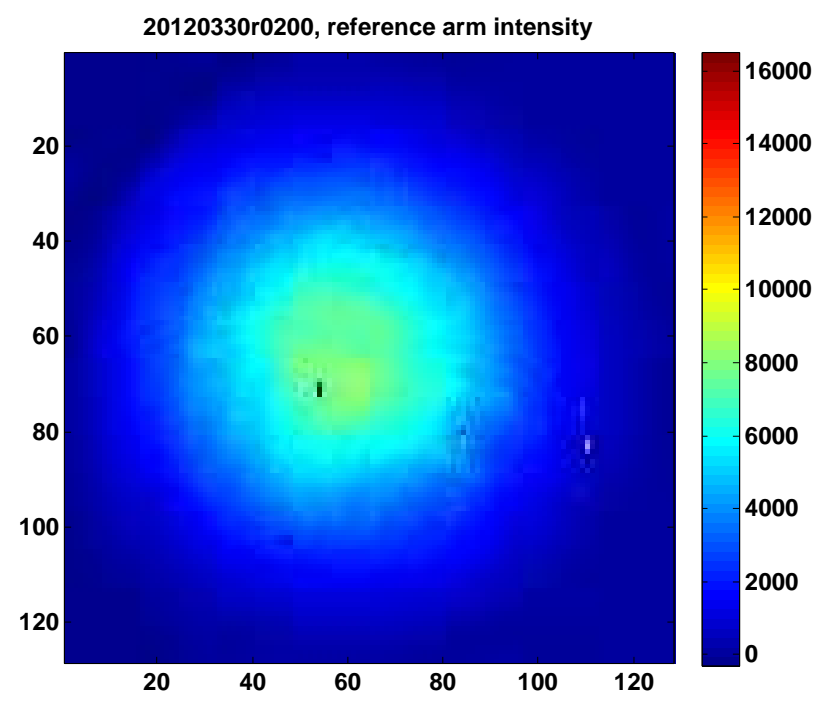

Figure 3. Intensity of reference arm measured when closing the shutter of the science beam. The intensity varies smoothly. The outliers features are due to bad pixels in the detector.

Inserting expressions (7) and (8) in to Eq. (3), we obtain the model relating phasor measurements and the input E-field at the DM pupil as

$$
\Phi(x, y) \approx t_{s} E_{0} A(x, y) \sqrt{I_{\mathrm{ref}}(x, y)} \exp \left[i \phi_{\mathrm{rs}}(x, y)+i \phi_{\mathrm{l}}(x, y)\right]\left(\epsilon_{\mathrm{h}}(x, y)+i \phi_{\mathrm{h}}(x, y)\right) .
$$

Note that we have neglected low spatial frequency portion of the post-coronagraph E-field because by design it is very much attenuated.

\section{CALIBRATION AND ALGORITHM DESCRIPTIONS}

\section{1 calibration}

In order to retrieve the wavefront aberrations $\phi_{\mathrm{h}}(x, y)$ and $\epsilon_{\mathrm{h}}(x, y 0$ at the DM pupil, it is necessary to calibrate $t_{s} E_{0} A(x, y), I_{\text {ref }}(x, y)$, and the low order phase $\phi_{\mathrm{rs}}(x, y)+\phi_{1}(x, y)$ in view of Eq. (9). The reference arm intensity $I_{\text {ref }}(x, y)$ can be readily measured by blocking the light from the science beam using the shutter. See Fig. 3. $t_{s} E_{0} A(x, y)$ can be measured by steering the beam away from the hole of the focal plane coronagraph mask to take a full image of the pupil because the full pupil intensity $I_{\text {full }}(x, y)$ is directly related to $t_{s} E_{0} A(x, y)$ via

$$
I_{\text {full }}(x, y)=\left|t_{s} E_{0} A(x, y)\right| .^{2} .
$$

Fig. 4 shows an example of the full pupil image where we can see the tapering effect of the pupil apodizer.

The low order phase $\phi_{\mathrm{rs}}(x, y)+\phi_{\mathrm{l}}(x, y)$ can be measured by introducing a sparse poking pattern on the DM, i.e. a known high spatial frequency wave pattern, and measuring the phasor response. Mathematically, we have

$$
\Delta \Phi(x, y) \approx i \sqrt{I_{\text {ref }}(x, y) I_{\text {full }}(x, y)} \exp \left[i\left(\phi_{\mathrm{rs}}(x, y)+\phi_{\mathrm{l}}(x, y)\right)\right] \Delta \phi_{\mathrm{DM}}(x, y)
$$

where $\Delta \phi_{\mathrm{DM}}$ is the sparse poking pattern and $\Delta \Phi(x, y)$ is the corresponding phasor change in response to the DM poking. It is convenient to rewrite Eq. (11) to have a pure imaginary quantity:

$$
\Delta \Phi(x, y) \exp \left[-i\left(\phi_{\mathrm{rs}}(x, y)+\phi_{\mathrm{l}}(x, y)\right)\right] \approx i \sqrt{I_{\mathrm{ref}}(x, y) I_{\mathrm{full}}(x, y)} \Delta \phi_{\mathrm{DM}}(x, y) .
$$




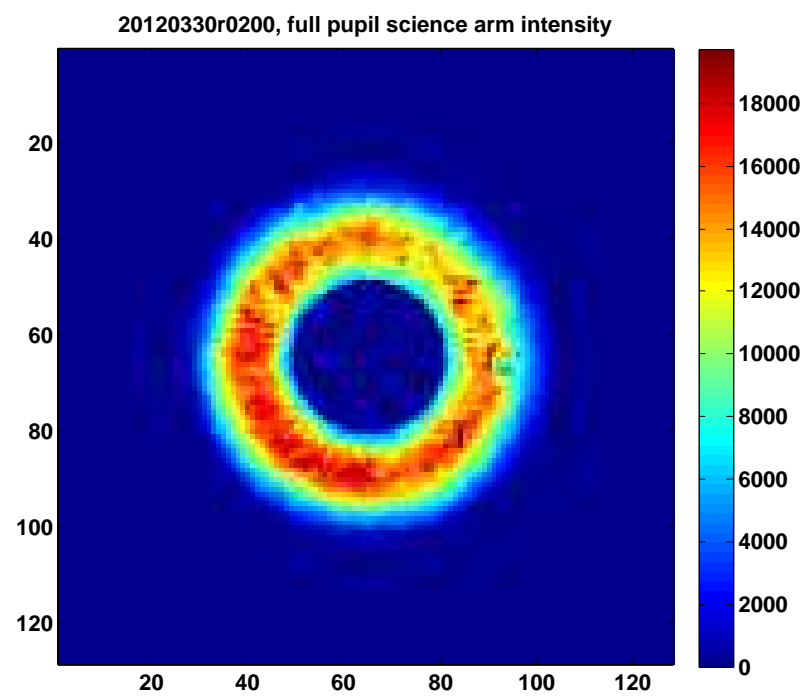

Figure 4. Full pupil image taken by steering the beam away from the hole at the center of the focal plane mask.

This enables us to estimate the low order phase $\phi_{\mathrm{rs}}(x, y)+\phi_{\mathrm{l}}(x, y)$. We parametrize the low order phase using the first $N=10$ Zernikes polynomials

$$
\phi_{\mathrm{rs}}(x, y)+\phi_{\mathrm{l}}(x, y)=\sum_{n=1}^{N} C_{n} Z_{n}(x, y),
$$

and solved $C_{n}$ 's to maximize the imaginary part

$$
\max _{C_{n}}\left\{\left|\Im\left[\Delta \Phi(x, y) \exp \left\{-i \sum_{n=1}^{N} C_{n} Z_{n}(x, y)\right\}\right]\right|^{2}\right\} .
$$

The left plot in Fig. 5 shows a sparse grid pattern that we used to poke the DM actuators. The DM poking pattern is a regular grid with spacing of $4 \mathrm{DM}$ actuators. We poke the DM in checkerboard fashion so that the adjacent DM actuators are poked in opposite directions to have a zero mean. This avoids an overall phase shift in the wavefront phase and thus has minimal effect on the reference beam. The imaginary part of the corresponding phasor response $\Delta \Phi(x, y) \exp \left[-i\left(\phi_{\mathrm{rs}}(x, y)+\phi_{\mathrm{l}}(x, y)\right)\right]$ is shown at the right side after we appropriately fit a low order phase.

Fig. 6 displays a phase map of the estimated low order phases. It is mainly a focus error with some tilt.

By relating the response pattern in the Calibrator pupil, with coordinate specified by the camera pixel row and column indices, and DM actuator poking pattern, specified at a (66x66) actuator grid, we register the DM actuators at the Calibrator pupil. This registration is described by an affine transformation,

$$
\left(\begin{array}{l}
x \\
y
\end{array}\right)=\left(\begin{array}{ll}
M_{11} & M_{12} \\
M_{21} & M_{22}
\end{array}\right)\left(\begin{array}{l}
x_{\mathrm{DM}} \\
y_{\mathrm{DM}}
\end{array}\right)+\left(\begin{array}{l}
x_{0} \\
y_{0}
\end{array}\right)
$$

where $(x, y)$ are indices in Calibrator pupil and $\left(x_{\mathrm{DM}}, y_{\mathrm{DM}}\right)$ labels actuator in DM pupil. The $2 \times 2$ matrix $M$ describes the optical imaging relation between the calibrator camera pupil and the DM pupil and $\left(x_{0}, y_{0}\right)$ is the coordinate of imaging point in Calibrator pupil of the origin of DM pupil. The transformation matrix $M$ and $\left(x_{0}, y_{0}\right)$ can be solved by fitting the grid patterns in Calibrator pupil to the DM poking pattern. For example, the transformation relating the patterns in the left and right plots in Fig. 5 is

$$
M=\left(\begin{array}{cc}
0.1452 & 1.2476 \\
1.2337 & -0.1460
\end{array}\right), \quad\left(\begin{array}{l}
x_{0} \\
y_{0}
\end{array}\right)=\left(\begin{array}{l}
18.2863 \\
28.6906
\end{array}\right) .
$$



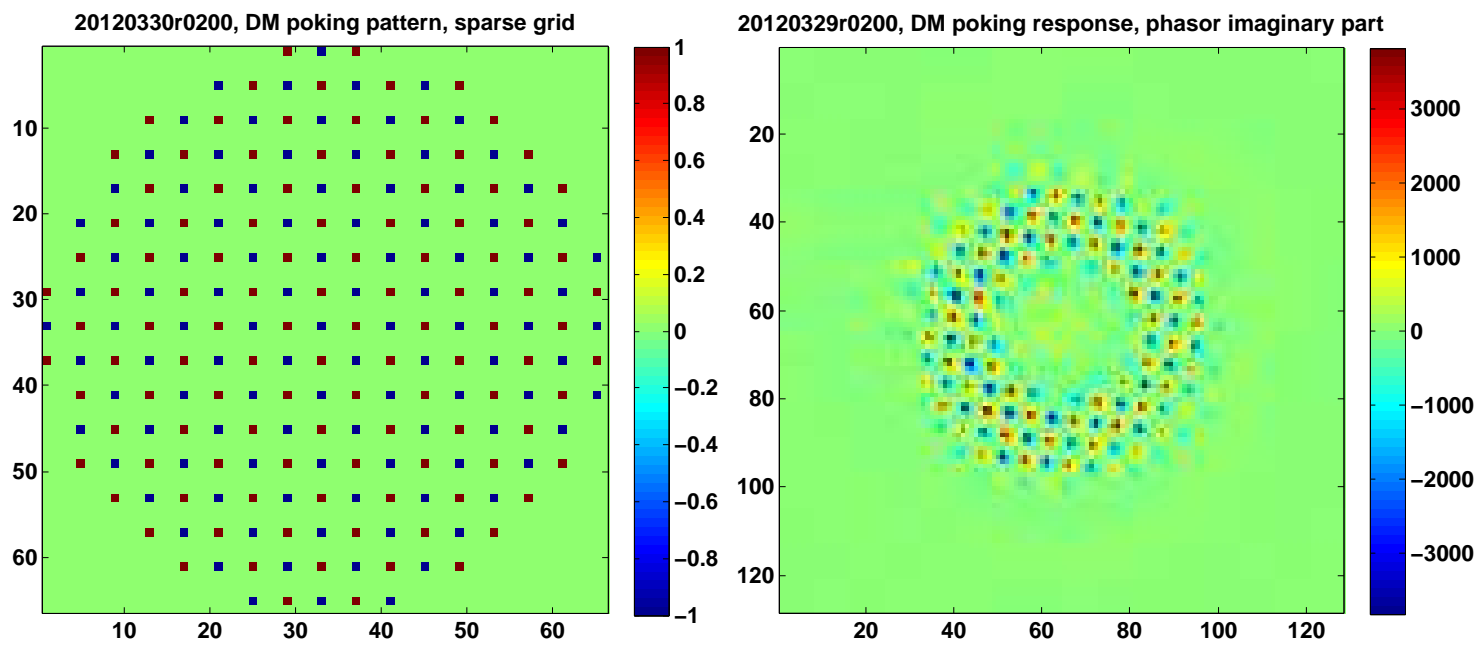

Figure 5. Sparse pattern used to poke the DM (left) and the corresponding phasor response (right) for calibrating the low order phase and the registration between DM actuators at the DM pupil and the camera pixels at Calibrator pupil.

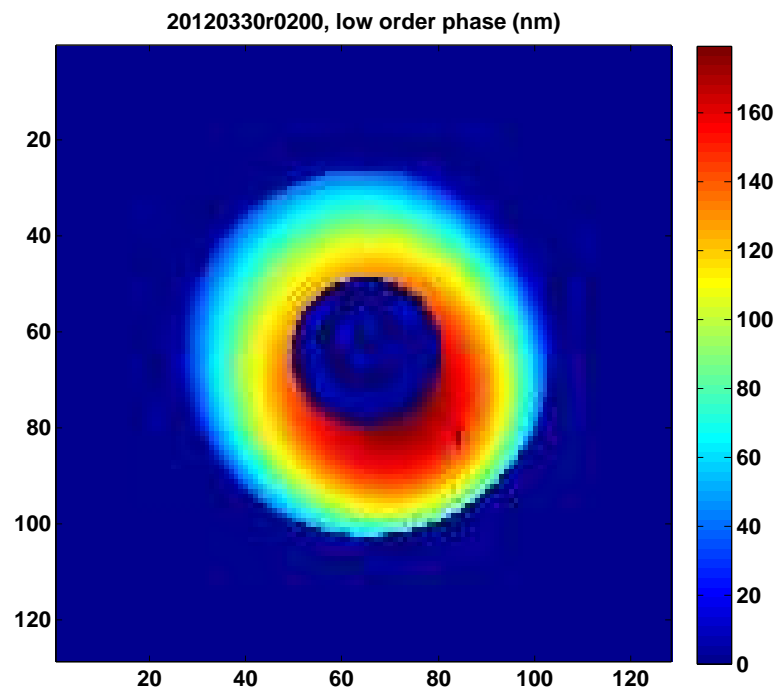

Figure 6. Estimated low order phase parametrized by the first 10 Zernikes polynomials. 
The fact that $M$ is dominated by the off diagonal terms means that the optical imaging between DM pupil and Calibrator pupil has a rotation of about 90 degree.

\subsection{Wavefront estimation}

With calibration measurements, we are ready to use the fringe measurements to estimate wavefront at the DM pupil. By design, the coronagraph focal plane mask attenuates the low order wavefront aberrations. Our first order wavefront estimation algorithm ignores the low order wavefront aberrations and concentrate on the high spatial frequency aberrations. Inverting (9), we get

$$
\epsilon_{\mathrm{h}}(x, y)+i \phi_{\mathrm{h}}(x, y) \approx \frac{\Phi(x, y)}{\sqrt{I_{\mathrm{ref}} I_{\mathrm{full}}(x, y)}} \exp \left\{-i \sum_{n=1}^{N} C_{n} Z_{n}(x, y)\right\},
$$

where we have used the estimated low order phases parametrized in Zernikes polynomials.

\subsection{Wavefront Correction}

With the knowledge of wavefront aberrations at the DM, we are ready to improve it. Nominally the AO system actively drives the wavefront at the $\mathrm{AO}$ wavefront sensor to a flat shape. In order to make any correction to this, we offset the DM actuator commands by an additional amount to correct any non-common path wavefront error as measured by the Calibrator. This correction is made iteratively. Let $\phi_{n}^{\mathrm{DM}-\text { offset }}(x, y)$ be the $\mathrm{n}$-th DM offset command. We have

$$
\phi_{n+1}^{\mathrm{DM}-\text { offset }}(x, y)=\phi_{n}^{\mathrm{DM}-\mathrm{offset}}(x, y)-g \hat{\phi}_{\mathrm{h}}(x, y)_{n}
$$

where $\hat{\phi}_{\mathrm{h}}(x, y)_{n}$ is estimated wavefront using Calibrator measurements for the $\mathrm{n}$-th iteration and $g$ is a gain parameter with value less than 1 . This forms an integral control loop.

\subsection{Half plane amplitude speckle cancellation}

Wavefront aberrations may be amplitude errors, which we do not have actuators to directly control. A residual amplitude error generates symmetric speckles in the image plane. It is possible to use a speckle canceling technique to cancel one of the paired speckles from the amplitude error while simultaneous brightening up the other speckle at the opposite side of the image plane. To illustrate this, let the amplitude error be a sine wave with spatial frequency being $\left(k_{x}^{0}, k_{y}^{0}\right)$,

$$
\epsilon_{\mathrm{h}}(x, y)=\epsilon_{0} \sin \left(k_{x}^{0} x+k_{y}^{0} y+\phi_{0}\right),
$$

where $\phi_{0}$ is a constant phase. In the image plane, this amplitude error leads to two speckles at $\left(k_{x}^{0}, k_{y}^{0}\right)$ and $\left(-k_{x}^{0},-k_{y}^{0}\right)$ symmetrically located about the center of the image plane as given by the Fourier transform of $\epsilon_{\mathrm{h}}(x, y)$ :

$$
\mathcal{F} \mathcal{T}\left\{\epsilon_{\mathrm{h}}(x, y)\right\}=\frac{\epsilon_{0}}{2 i}\left[e^{i \phi_{0}} \delta\left(k_{x}-k_{x}^{0}\right) \delta\left(k_{y}-k_{y}^{0}\right)-e^{-i \phi_{0}} \delta\left(k_{x}+k_{x}^{0}\right) \delta\left(k_{y}+k_{y}^{0}\right)\right] .
$$

To cancel the speckle at $\left(k_{x}^{0}, k_{y}^{0}\right)$, we introduce a phase error of the same spatial frequency, but with a pi/2 phase shift,

$$
\phi_{\mathrm{h}}(x, y)=\epsilon_{0} \sin \left(k_{x}^{0} x+k_{y}^{0} y+\phi_{0}+\pi / 2\right)=\epsilon_{0} \cos \left(k_{x}^{0} x+k_{y}^{0} y+\phi_{0}\right),
$$

so that the total E-field may be expressed as

$$
\epsilon_{\mathrm{h}}(x, y)+i \phi_{\mathrm{h}}(x, y)=i \epsilon_{0} \exp \left[-i\left(k_{x}^{0} x+k_{y}^{0} y+\phi_{0}\right)\right],
$$

which is of a single frequency at $\left(-k_{x}^{0},-k_{y}^{0}\right)$. This means that we only have one speckle at $\left(-k_{x}^{0},-k_{y}^{0}\right)$ which is four times brighter as its amplitude is twice of that from a sine wave. In this way, we can cancel speckles at a half plane to make one half of the image plane darker while the other half brighter. 

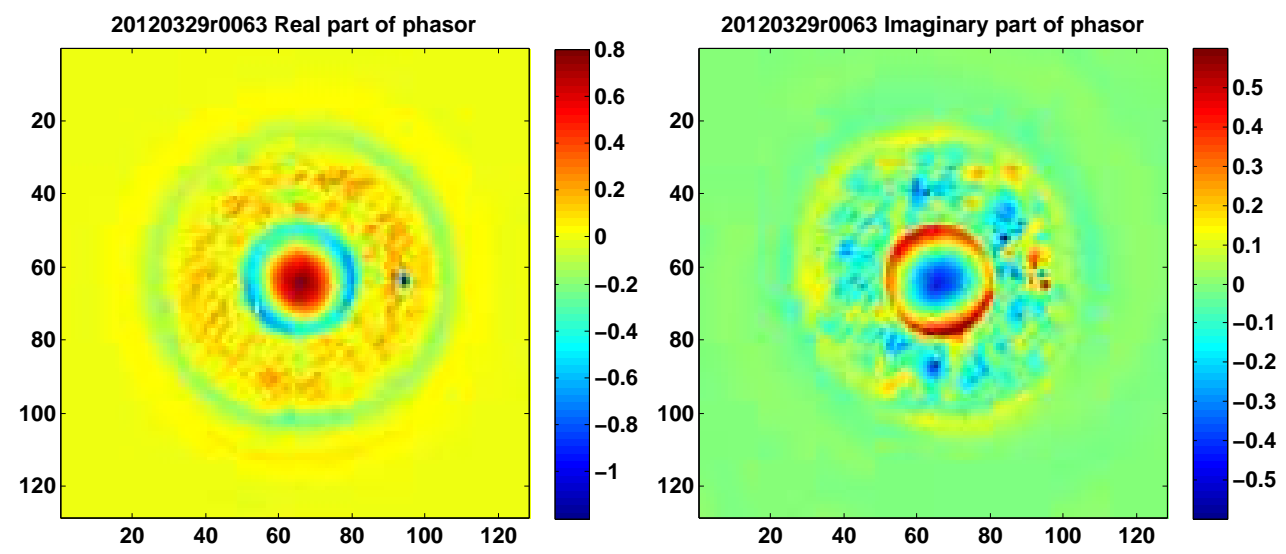

Figure 7. Real (left) and imaginary (right) parts of the phasor map. They are normalized using the average intensity over the pupil.
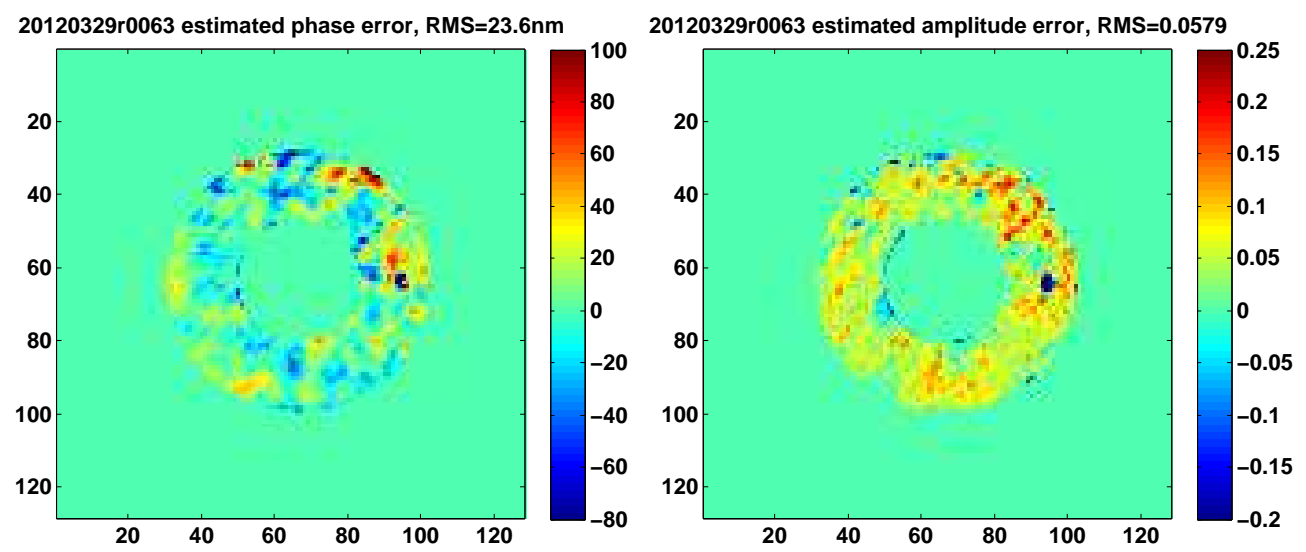

Figure 8. An estimated initial wavefront at the DM pupil before any attempt to correct it. Phase map is at the left and amplitude is at the right.

\section{RESULTS}

In this section, we present some results from our recent experiments. The primary measurements of P1640 Calibrator are the ABCD fringe frames. The intensity variations between these four frames give the phasors as defined in Eq. (3). Fig. 7 displays the real and imaginary parts of the measured phasors. The original phasors usually carry an overall tilt, a linear phase trend across the pupil. Here we have detrended this linear phase trend. Note that due to low order phase $\phi_{\mathrm{rs}}(x, y)+\phi_{\mathrm{l}}(x, y)$ in Eq. (9), we can not identify the real and imaginary parts of the phasors as the map of amplitude and phase error maps.

Using Eq. (17), we can estimate the high order wavefront at the DM pupil. Fig. 8 shows maps of estimated wavefront phase and amplitude before we use DM to do any correction. The initial phase error has $23 n m$ RMS with about 0.06-0.07 RMS relative amplitude errors. As described in subsection 3.3, we can iteratively feed wavefront correction command back to the DM according to the estimated wavefront. The correction adds an offset to the DM control loop to compensate the non-common path wavefront errors. Fig. 9 shows an improved wavefront after more than 10 iterations with a gain of 0.5. The wavefront phase error RMS is reduced to 10nm while the amplitude does not have much change.* This shows that P1640 Calibrator properly senses the wavefront; both the wavefront estimation algorithm and feedback loop are effective.

\footnotetext{
${ }^{*}$ Because P1640 Calibrator measures broadband fringes, as we drift away from the central fringe envelop, the visibility drops. This may account for the smaller RMS values of the amplitude after the DM wavefront corrections.
} 
20120329 r0092 estimated phase error, RMS $=10.5 \mathrm{~nm}$

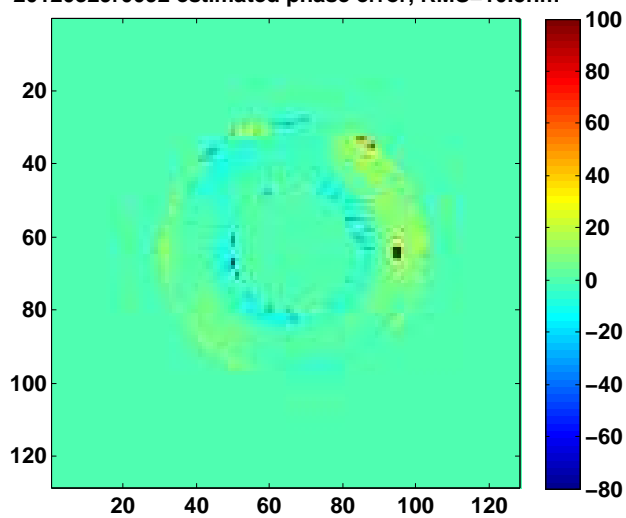

20120329 r0092 estimated amplitude error, RMS $=0.0518$

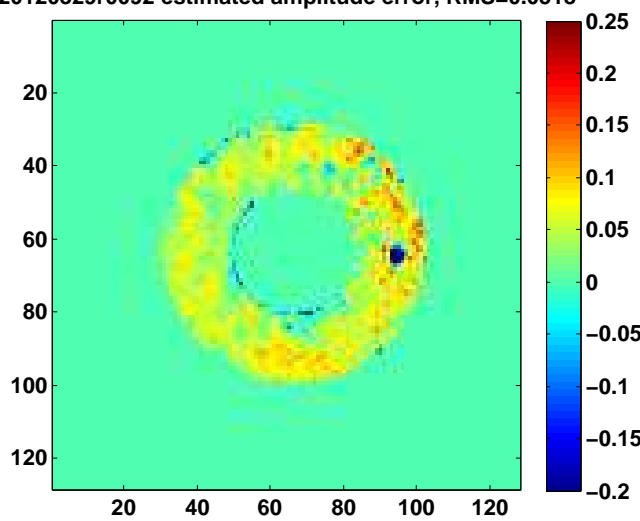

Figure 9. Phase (left) and amplitude (right) maps at the DM pupil after improvements.
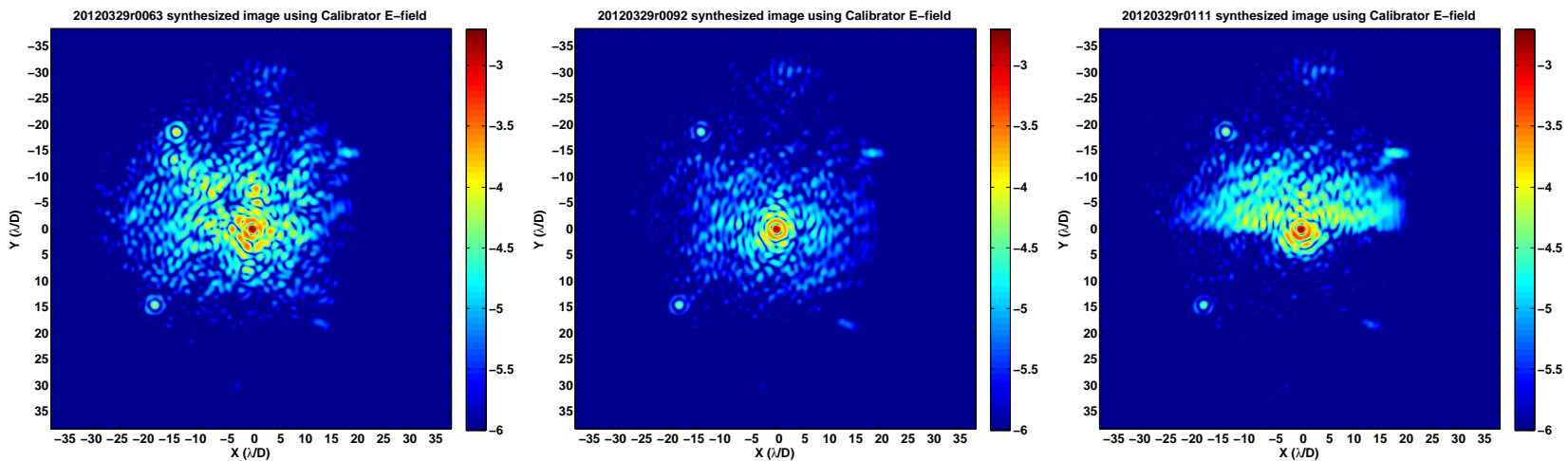

Figure 10. Synthesized images using E-field measured by the Calibrator, before correction using Calibrator measurements (left), after phase conjugation using Calibrator data (center), and the half plane speckle nulling (right)

Fig. 10 shows the synthesized images using the E-field measured by the Calibrator and propagating it to the image plane. These are the predictions of images from the Calibrator measurements with the left plot showing the image for the initial wavefront shown in Fig. 8 and the center plot showing the image for the improved wavefront as shown in Fig. 9. It is not hard to see the reduced speckles in the center plot compared with the left one. Because P1640 calibrator measures both amplitude and phase errors, using the speckle nulling technique described in subsection 3.4, we offset the DM and canceled the speckles due to the amplitude errors achieving a half image plane dark region clearly shown in the right plot in Fig. 10. This also shows that after our phase correction, the speckles are mainly due to the amplitude errors.

To show the reduced speckle intensity level, Fig. 11 displays the intensity radial distributions computed by taking an azimuth average of the synthesized images shown in Fig. 10. The three curves represent respectively the intensity level radial distributions for three cases of before the correction using P1640 Calibrator measurements (red), after the correction using P1640 Calibrator measurements (black), and the dark side of the half plane speckle canceling (blue). Fig. 12 shows the images taken by the science camera (IFS, integral field spectrograph) before and after phase corrections using P1640 Calibrator measured wavefronts. Fig. 12 displays two images taken by the IFS on sky during science observation of an A star. The left and right images are from the channel at $1.2 \mu \mathrm{m}$ wavelength corresponding to before and after the wavefront corrections using P1640 Calibrator wavefront measurements. With the wavefront improvement using P1640 Calibrator, in the right plot, we can see a square shape dark region for the first time. This dark region has angular size of $64 \lambda / D$, corresponding to the the control bandwidth $[-32,32] \lambda / D$ of the P3K AO system. 


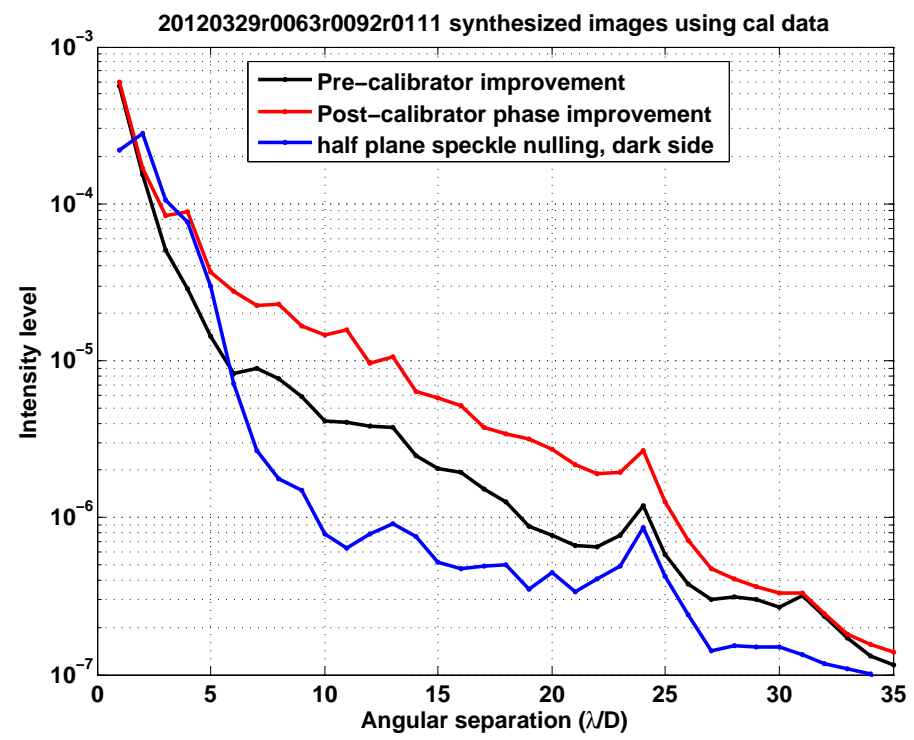

Figure 11. Azimuthly averaged intensity level distribution along radial direction.

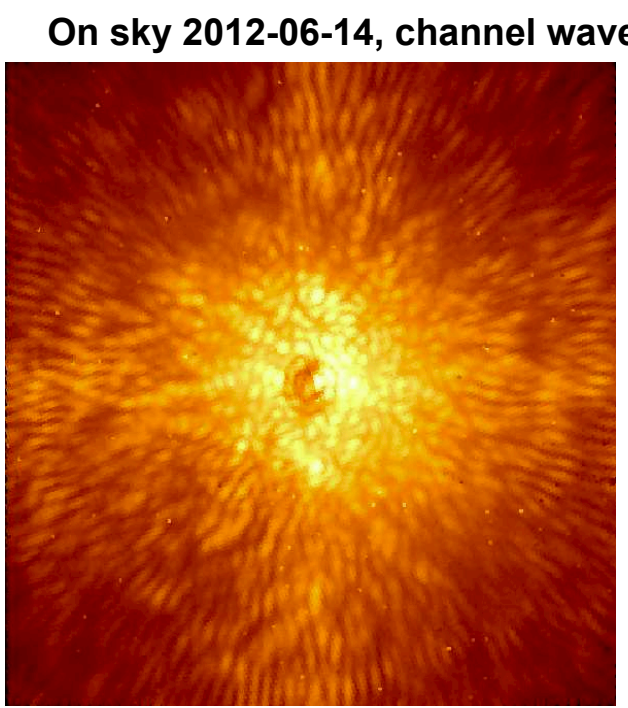

Before calibrator correction

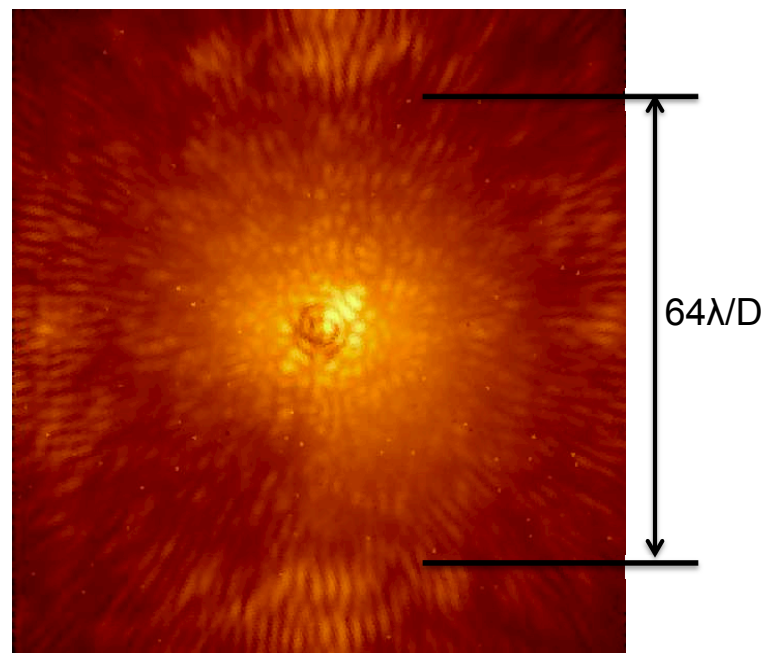

After calibrator correction

Figure 12. On sky images taken by the IFS while observing an A star. Left image is before the wavefront correction the right image is after the correction. 


\section{CONCLUSIONS, DISCUSSIONS, AND FUTURE WORKS}

We have demonstrated that the P1640 Calibrator measures high spatial frequency wavefront properly. The efficacy is demonstrated by observing the improvements in the wavefronts after feeding the derived wavefront correction back to the DM. We are also able to achieve a half plane dark region by adopting a speckle canceling technique to remove the speckles due to amplitude errors. The IFS images ultimately show the quality improvements in the high contrast images after applying the P1640 Calibrator corrections.

Even though the coronagraph is designed for passing E-field of high spatial frequency, the cutoff is not a step function. Low spatial frequency aberrations can still leak through the system and cause extra light to be at the image plane. This means that P1640 Calibrator can measure low order wavefront with some degradation in sensitivity. Working out the algorithm is one subject of our future works.

Using a speckle canceling technique, we were able to achieve a half image plane dark region clearly shown in the synthesized image. Due to the Lyot pupil mask, the actual IFS, however, does not have a well defined dark region. The differences in the speckle patterns between the synthesized images and the IFS images come from the extra optics between the beam splitter and the science instrument, including the Lyot coronagraph pupil mask which contains a spider structure. Bridging the gap between synthesized images and the IFS images forms another topic of our future works.

Finally, our performance is not completely limited by noise yet. We are working toward a higher contrast ratio by reducing systematic errors.

\section{ACKNOWLEDGMENTS}

This work was prepared at the Jet Propulsion Laboratory, California Institute of Technology, under a contract with the National Aeronautics and Space Administration.

\section{REFERENCES}

[1] Sivaramakrishana, A., Soummer, R., Pueyo, L., Wallace, J. K., and Shao, M., "Sensing phase aberrations behind lyot coronagraphs," Ap.J. 688, 701-708 (2008).

[2] Macintosh, B. A., Graham, J. R., Palmer, D. W., Doyon, R., Dunn, J., Gavel, D., Larkin, J., Oppenheimer, B., Saddlemyer, L., Sivaramakrishnan, A., Wallace, J. K., Bauman, B., Erickson, D. A., Marois, C., Poyneer, L. A., and Soummer, R., "The gemini planet imager: from science to design to construction," Proc. SPIE 7015, 100-112 (2008).

[3] Hinkley, S., Oppenheimer, B. R., Zimmerman, N., Brenner, D., Parry, I. R., Crepp, J. R., Vasisht, G., Ligon, E., King, D., Soummer, R., Sivaramakrishnan, A., C. Beichman, M. S., Roberts, L. C., Bouchez, A., Dekany, R., Pueyo, L., Roberts, J. E., T. Lockhart, C. Z., Shelton, C., and Burruss, R., "New high contrast imaging program at palomar observatory," Publications of the Astronomical Society of the Pacific. 123, 76-86 (2011).

[4] Openheimer, B. R., Beichman, C., Brenner, D., Burruss, R., Cady, E., Crepp, J., Hillenbrand, L., Hinkley, S., Ligon, E. R., Lockart, T., Parry, I., Pueyo, L., Rice, E., Roberts, L. C., Roberts, J., Shao, M., Sivaramakrishnan, A., Soummer, R., Vasisht, G., Vescelus, F., Wallace, J. K., Zhai, C., and Zimmerman, N., "Project 1640: the world's first exao coronagraphic hyperspectral imager for comparative planetary science," Proc. SPIE This volumne, TBD (2012).

[5] Soummer, R., "Apodized pupil lyot coronagraphs for arbitrary telescope apertures," Ap.J. 618, L161 (2005).

[6] Colavita, M. M., Wallace, J. K., Hines, B. E., Gursel, Y., Malbet, F., Palmer, D. L., Pan, X. P., Shao, M., Yu, J. W., Boden, A. F., Dumont, P. J., Gubler, J., Koresko, C. D., Rulkarni, S. R., Lane, B. F., Mobely, D. W., and van Belle, G. T., "The palomar testbed interferometer," Astrophys. J. 510, 505-521 (1999). 